\title{
The Anti Matter Spectrometer (AMS-02): a particle physics detector in space
}

\author{
R. Battiston on behalf of the AMS Collaboration \\ Physics Department and INFN Section, Perugia, 06123, Italy \\ Presenter: Roberto Battiston (r.battiston@tiscali.it)
}

AMS-02 is a space borne magnetic spectrometer designed to measure with accuracies up to one part in $10^{9}$ the composition of Cosmic Rays near Earth. With a large acceptance $\left(5000 \mathrm{~cm}^{2} \mathrm{sr}\right)$, an intense magnetic field from a superconducting magnet $(0.7 \mathrm{~T})$ and an accurate particle identifications AMS-02 will provide the highest accuracy in Cosmic Rays measurements up to the TeV region. During a three years long mission on the ISS AMS-02 will achieve a sensitivity to the existence of anti-He in the Cosmic Rays of one part in a billion as well as important informations on the origin of Dark Matter. We review the status of the construction of the AMS-02 experiment in preparation for the three years mission on the ISS.

\section{Introduction}

AMS is a particle physics experiment in space. The purpose is to perform accurate, high statistics, long duration measurements of the spectra of energetic (up to multi-TeV) primary cosmic rays in space. Some of the physics goals are:

- Dark Matter: There are many theoretical suggestions [1] that particles predicted by SUSY theories, for example the neutralino $\chi$, are a component of the Dark Matter which constitutes one quarter of the mass of the universe. Collisions of dark matter in the galactic halo produce $\overline{\mathrm{p}}, \mathrm{e}^{+}$and $\gamma$ via:

$$
\begin{aligned}
\chi+\chi & \rightarrow \overline{\mathrm{p}}+\ldots \\
& \rightarrow \mathrm{e}^{+}+\ldots \\
& \rightarrow \gamma+\ldots
\end{aligned}
$$

The $\overline{\mathrm{p}}, \mathrm{e}^{+}$and $\gamma$ from these collisions will produce deviations from the smooth energy spectra. Therefore, the precision measurement of the $\overline{\mathrm{p}}, \mathrm{e}^{+}$and $\gamma$ spectra will enable AMS to establish whether SUSY particles are the origin of Dark Matter. There are also predictions that antideuterons can be produced from the collision of SUSY particles, at a level which AMS could detect [2].

- Antimatter: The strong evidence which supports the Big Bang theory of the origin of the universe requires matter and antimatter to be equally abundant at the very hot beginning [3]. The absence of sharp annihilation $\gamma$ ray peaks excludes the presence of large quantities of antimatter within our local super cluster of galaxies. However there is no direct experimental evidence regarding the remainder $\left(10^{8}\right)$ of the universe.

- Cosmic rays: AMS will collect $\sim 10^{9}$ nuclei and isotopes (D, He, $\mathrm{Li}, \mathrm{Be}, \mathrm{B}, \mathrm{C} \ldots \mathrm{Fe}$ ). Among the interesting issues which will be studied are the accurate determination of the ratio of boron to carbon over a wide range of energies provides crucial information regarding the propagation of cosmic rays in the galaxy. In particular, the ratio of ${ }^{10} \mathrm{Be}$ (mean lifetime of $3.6 \times 10^{6}$ years) to the stable ${ }^{9} \mathrm{Be}$ will enable us to extend the low-energy measurements of the Ulysses satellite to higher energies and to provide important information on the understanding of cosmic ray propagation. 
To perform a high accuracy measurement of the spectra of energetic charged particles in space, the AMS detector is based on experience and observations from experiments to study rare signals among intense backgrounds, such as the study of leptonic decays of vector mesons from $\gamma+\mathrm{N} \rightarrow \mathrm{N}+\left(\rho, \omega, \phi \rightarrow \mathrm{e}^{+} \mathrm{e}^{-}\right)$at DESY [4] and the discovery of the $\mathrm{J}$ particle from $\mathrm{p}+\mathrm{N} \rightarrow \mathrm{J}\left(\rightarrow \mathrm{e}^{+} \mathrm{e}^{-}\right)+\ldots$ [5] combined with the precision measurements made in the study of $\mathrm{Z}$ decays [6]. These experiments were successful because they have:

(a) Minimal material in the particle trajectory so that the material itself is not a source of background nor a source of large angle nuclear scattering;

(b) Many repeated measurements of momentum and velocity so as to ensure that background particles which had experienced large angle nuclear scattering from the detector itself be swept away by the spectrometer and not confused with the signal.

It was the strict adherence to these techniques that ensured that a background rejection of $10^{10}$ was indeed possible and made these experiments successful. AMS is designed following the same principles. Figure 1 shows the AMS-02 detector configuration for the International Space Station (ISS).

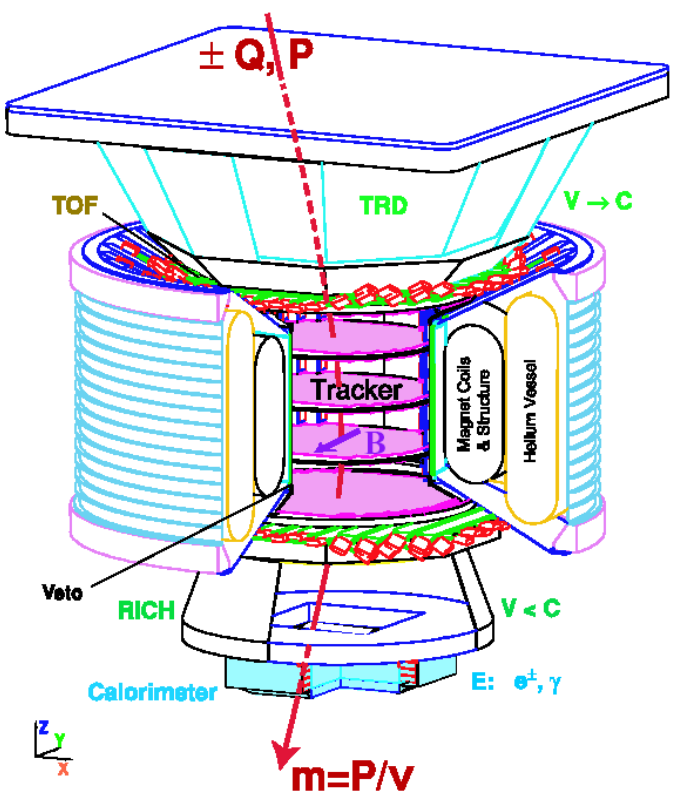

Figure 1: A TeV Detector in Space: AMS-02 on the Space Station

AMS-02 will contain the following main components:

(1) A twenty layer Transition Radiation Detector (TRD) which identifies electrons and positrons with a measured rejection factor against hadrons of $10^{3}$ to $10^{2}$ from $1.5 \mathrm{GeV}$ to $300 \mathrm{GeV}$.

(2) Four layers of Time of Flight (TOF) hodoscopes that provide precision time of flight measurements $(\sim 120$ picoseconds) and $\mathrm{dE} / \mathrm{dX}$ measurements.

(3) The superconducting magnet, which provides a bending power of $\mathrm{BL}^{2}=0.86 \mathrm{Tm}^{2}$. 
(4) Eight layers $\left(6.45 \mathrm{~m}^{2}\right)$ of silicon tracker, which provide a proton rigidity (= momentum/charge) resolution of $20 \%$ at $0.5 \mathrm{TV}$ and a helium $(\mathrm{He})$ resolution of $20 \%$ at $1 \mathrm{TV}$ and charge resolution of nuclei up to iron $(Z=26)$.

(5) Veto, or anticoincidence, counters (ACC) which ensure that only particles passing through the magnet aperture will be accepted.

(6) A Ring Imaging Cerenkov Counter (RICH), which measures the velocity (to $0.1 \%$ ) and charge $|\mathrm{Z}|$ of particles or nuclei. This information, together with the measurement of momentum in the tracker, will enable AMS to unambiguously determine the mass of these particles and nuclei.

(7) A 3-D sampling calorimeter (ECAL) made out of $16.7 \mathrm{X}_{\mathrm{o}}$ (radiation lengths) of lead and scintillating fibers which measures the energy of gamma rays, electrons and positrons and distinguishes electrons and positrons from hadrons with a rejection of $10^{4}$ in the range between 1.5 $\mathrm{GeV}$ to $1 \mathrm{TeV}$.

(8) A system of two star trackers (AMICA) to allow the precise reconstruction of the origin of high energy gamma rays reconstructed in the detector.

Due to the limits in the available space, in the following sections we will discuss the characteristics of only a few among the main subsystems of the AMS-02 apparatus as well as the status of their construction and space qualification.

\section{The Cryomagnet}

The purpose of the superconducting magnet is to extend the energy range of our measurements of particles and nuclei to the multi-TeV region [7]. The magnet design was based on the following technical considerations:

(i) Experience in designing and manufacturing the AMS-01 magnet which was 10 times safer than stress limits allowed.

(ii) The result of many years of intensive $R \& D$ collaboration to design a magnet with the following properties :

- Identical field configuration to the AMS-01 magnet to maintain mechanical stability and follow NASA safety standards;

- Minimized heat loss $(\sim 100 \mathrm{~mW})$ and minimized quench probability;

(iii) We have chosen to have the magnet built by experts from the Oxford Instruments R\&D group, who has an excellent record to produce highly reliable magnets running in persistent mode without quench. This group has produced the 8 OSCAR magnets (2.36 Tesla) used in cyclotrons in Japan and England and which have operated for close to 30 years without any quench. This group also built the CLEO magnet at CORNELL and the CLAS torus at Jefferson Laboratory and the KLOE magnet in Frascati. All are large, high-field, special-purpose magnets which have operated for years without quench. To ensure that these experts are able to devote all their efforts to the construction of the AMS-02 magnet, we have supported a new company: Space Cryomagnetics Ltd., entirely staffed by the experts of the Oxford Instruments R\&D group and entirely dedicated to the AMS-02 magnet.

Two magnets are being built. One is the flight magnet and the other is used for space qualification tests. The magnet has no magnetic field during the shuttle launch and landing and so there is no force among the coils, hence for the test magnet the coils are replaced by mass equivalents.

The magnet system, as shown in Figure 2, consists of superconducting coils, a superfluid helium vessel and a cryogenic system, all enclosed in a vacuum tank. Outside of the vacuum tank are supporting electronics, located in the Cryomagnet Avionics Box (CAB), valves and cabling. The vacuum tank is toroidal with inner 
diameter of $1.1 \mathrm{~m}$, outer diameter of $2.7 \mathrm{~m}$ and a length of the central cylinder surrounding the tracker of 0.9 $\mathrm{m}$. The magnet operates at a temperature of $1.8 \mathrm{~K}$, cooled by superfluid helium stored in the vessel. It is launched at the operating temperature, with the vessel full of 2500 litres of superfluid helium. The magnet will be launched with no field, it will be charged only after installation on the ISS. Because of parasitic heat loads, the helium will gradually boil away throughout the lifetime of the experiment. After the project time of 3 to 5 years, the helium will be used up and the magnet will warm up and no longer be operable.

The coil system consists of a set of 14 superconducting coils arranged, as shown in Figure 2, around the inner cylinder of the vacuum tank. The coil set has been designed to give the maximum field in the appropriate direction inside the bore tube, while minimising the stray field outside the magnet.

The single large pair of coils generates the magnetic dipole field perpendicular to the experiment axis. The twelve smaller flux return coils control the stray field and, with this geometry, they also contribute to the useful dipole field. The magnetic flux density at the geometric centre of the system is $0.86 \mathrm{~T}$. Table 1 summarizes some of the magnet parameters.

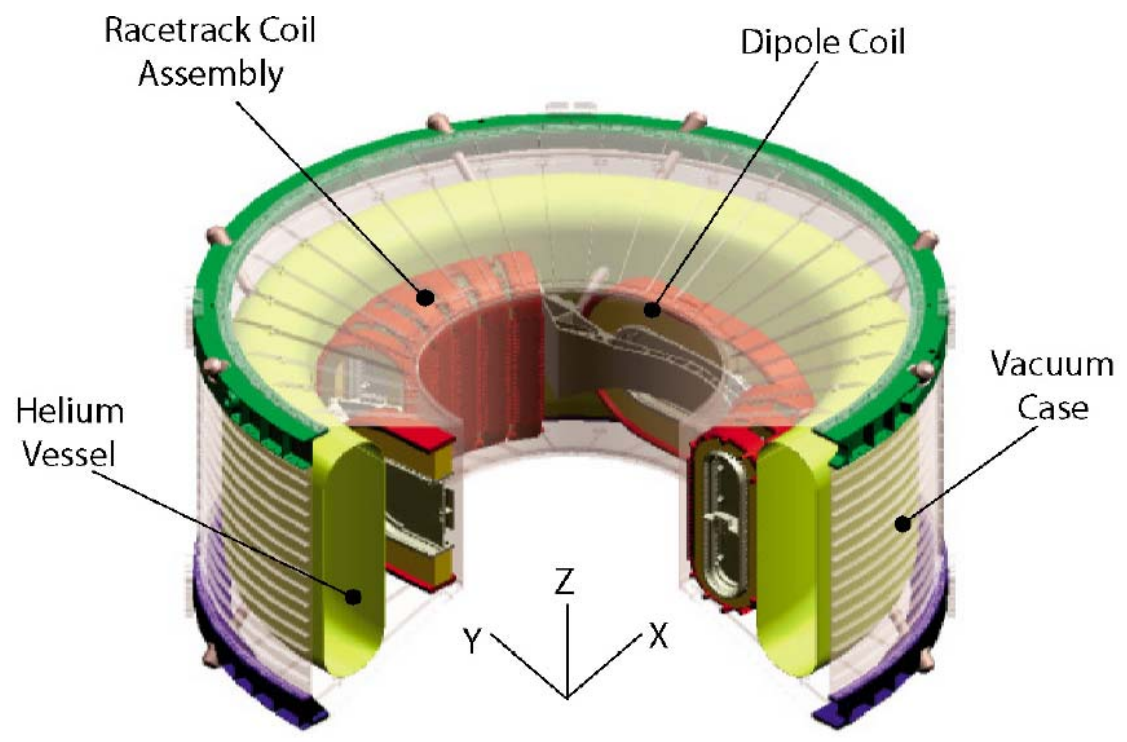

Figure 2: AMS-02 superconducting magnet layout.

Table 1 AMS-02 Cryomagnet parameters

\begin{tabular}{|l|c|}
\hline Central Magnetic Field Bx $(0,0)[\mathrm{T}]$ & 0.860 \\
\hline Dipole Bending Power [Tm $\left.{ }^{2}\right]$ & 0.862 \\
\hline Maximum Stray Magnetic Field at R=2.3 m [mT] & 15.2 \\
\hline Maximum Stray Magnetic Field at $\mathrm{Y}=2.3 \mathrm{~m}[\mathrm{mT}]$ & 7.6 \\
\hline Maximum Stray Magnetic Field at R=3.0 m [mT] & 3.9 \\
\hline Peak Magnetic Field on the Dipole Coils [T] & 6.59 \\
\hline Peak Magnetic Field on the Racetrack Coils [T] & 5.91 \\
\hline Maximum Torque in geomagnetic field [Nm] & 0.272 \\
\hline Nominal Operating Magnet Current [A] & 459.5 \\
\hline Stord Energy [MJ] & 5.15 \\
\hline Nominal Magnet Inductance [H] & 48 \\
\hline
\end{tabular}


The superconducting wire was developed specifically to meet the requirements of the AMS cryomagnet [1]. The current is carried by tiny $(22.4 \mu \mathrm{m}$ diameter) filaments of niobium titanium (NbTi) which - given the magnetic flux and current densities within the coils - carries the current without resistance provided the temperature is kept below $4.0 \mathrm{~K}$.

Because pure $\mathrm{NbTi}$ has rather low thermal conductivity, it is prone to instability. This can be overcome if it is in intimate contact with a material which has a high thermal conductivity at the cryogenic operating temperature, such as a pure metal. For this reason, the NbTi filaments are embedded in a copper matrix, which is encased in high-purity aluminium. The copper is required for manufacturing reasons, but the aluminium is extremely conductive and much less dense, thus providing maximum thermal stability for aluminium weight. The diameter of the round copper strand at the centre is $0.76 \mathrm{~mm}$, and the aluminium dimensions are $2.0 \mathrm{~mm} \times 1.5 \mathrm{~mm}$. The current density in the superconductor is $2300 \mathrm{~A} / \mathrm{mm}^{2}$ or $157 \mathrm{~A} / \mathrm{mm}^{2}$ including the aluminium.

To manufacture the coils, the superconducting wire was first cleaned then insulated using $85 \mu \mathrm{m}$ thick polyester tape. Each coil was wound separately onto an aluminium former from a single length of conductor before being impregnated under vacuum with epoxy resin. The impregnation process gives the coils mechanical integrity, and also provides electrical insulation between turns and layers. After completion, each coil is tested individually under conditions as representative as possible of flight, to test the integrity of the design and the quality of the build.

Each of the two larger (dipole) coils, which generate most of the useful field, has 3360 turns, and the 12 smaller (flux return) coils each have 1457 turns. The 14 coils are connected in series, with a single conductor joint between each pair of adjacent coils. The current when the magnet is operating is $459.5 \mathrm{~A}$. All of the coils have been manufactured and tested (see Figure 3).

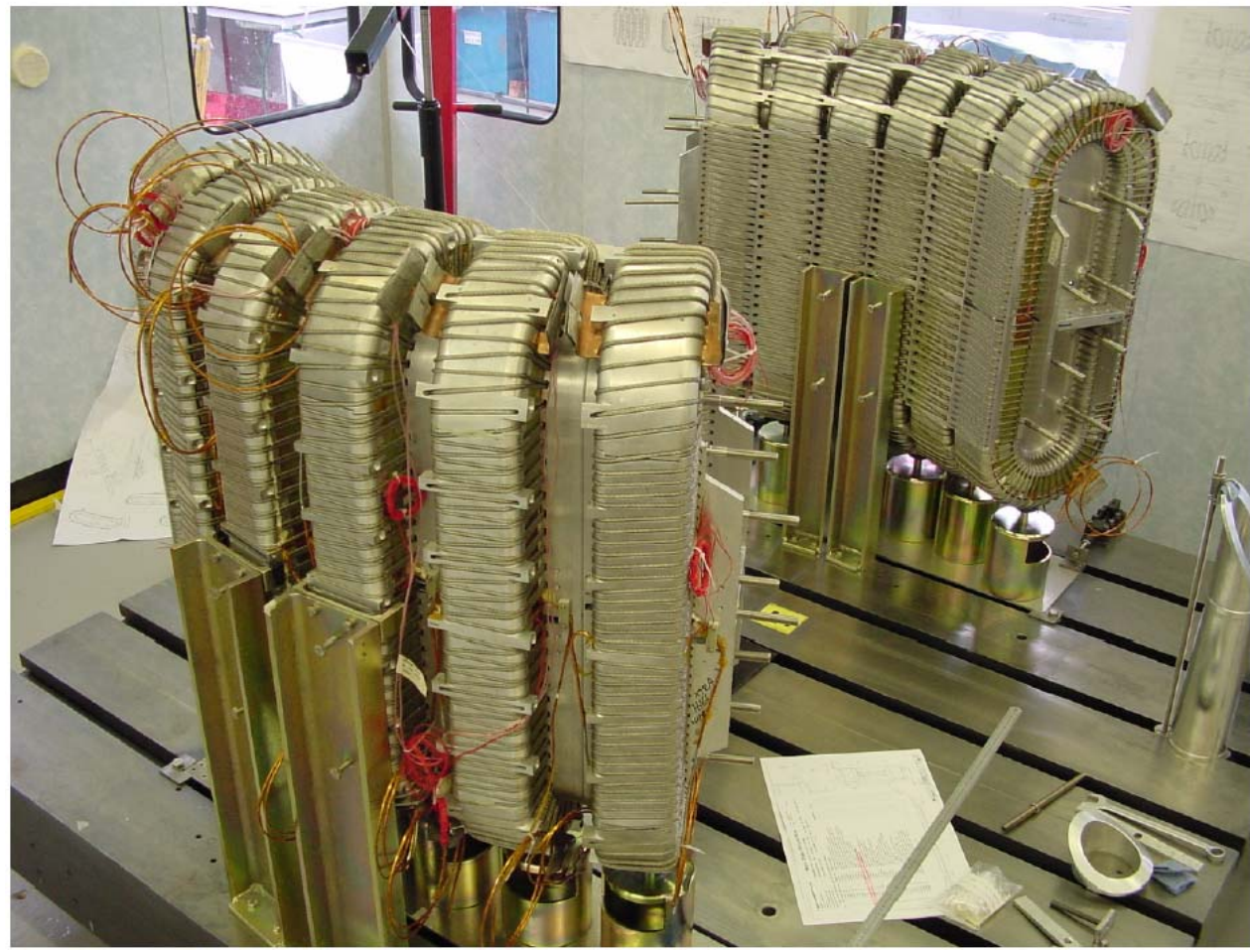

Figure 3: Coil Assembly 
The coils are not coupled thermally. This means that a quench in one coil, leading to a rise in temperature, will not necessarily propagate to any of the others. If this was allowed to happen, the entire stored energy of the magnet $(5 \mathrm{MJ})$ could be dissipated as heat in the coil which quenched. While the temperatures reached in that coil would not be dangerous, sufficient thermal stresses could be induced (by differential thermal contraction between parts of the coil) that the performance of the coil might be permanently reduced. For this reason, all the coils are constantly monitored by an electronic protection system. If the onset of a quench is detected in any coil, heaters are powered in the other coils to quench all 14 simultaneously. This distributes the stored energy between the coils, preventing any single coil from taking a disproportionate amount of energy which could otherwise result in degradation. The operation of these quench heaters is an important part of the testing and qualification procedure for the magnet coils.

Each coil is subject to internal forces as a result of its own magnetic field. In general, these are burst forces, trying to expand the racetrack-shaped coil into a ring. These loads are in the plane of the coil, and are resisted by the former on which the coil was wound. In addition, each coil is attracted or repelled by all the other coils in the magnet. This leads to a relatively complicated load system on some of the coils, with forces perpendicular to the plane of the coil. The magnetic loads are quite large: the two dipole coils feel a net attraction to each other of around 250 tonnes. During individual coil testing, each coil is charged until some part of the winding is subject to the same force it will experience in flight.

The cold mass of the magnet is more than $2000 \mathrm{~kg}$. This has to be supported from the experiment structure (in particular the vacuum tank), which is at ambient temperature $(\sim 270 \pm 40 \mathrm{~K})$. The design of the support straps is therefore crucial, as they have to be able to carry the load without conducting significant heat across the large temperature gradient. Each consists of a pair of composite bands connected in parallel. One band is thin, with low stiffness and strength, and is permanently connected between the cold mass and the vacuum tank. The other band is much thicker and stronger, but possesses a passive disconnect feature. This means that it only forms a thermal path between the vacuum tank and the magnet during launch and landing. At other times (when it is not needed), differential thermal contraction between the bands and the removal of the high inertial load cause the disconnect to open, dramatically reducing the thermal conduction of the support. A total of 16 straps support the magnet from the vacuum tank. During normal operations on the ground or in space, only the low-stiffness band is engaged, and the heat conduction is very low (less than 3 $\mathrm{mW}$ per support). During launch, the high-stiffness band engages as well. The conducted heat load is much higher but because the launch takes only a few minutes the effect on the overall endurance of the system is not significant. The dual stiffness characteristic of the straps makes their behaviour non-linear and, as major structural components of the magnet system, they have been subject to special scrutiny and testing, particularly testing to failure to understand the safety margins.

The cooling of terrestrial superconducting magnets using liquid helium is a well-established technology, but there is much less experience of helium cryogenics in space. The cryogenic system for the AMS magnet combines technologies from terrestrial magnet cryogenics and space cryogenics to meet the particular challenges of the Space Shuttle launch and the environment of the ISS [8]. It maintains the magnet at a temperature of $1.8 \mathrm{~K}$, under all operating conditions, for the duration of the experiment. Liquid helium $\left({ }^{4} \mathrm{He}\right)$ can exist in two forms. Normal liquid helium behaves in a conventional manner. But if it is cooled below $2.17 \mathrm{~K}$, some of its properties change dramatically as it becomes a superfluid. In particular, its viscosity falls almost to zero, and its apparent thermal conductivity increases by many orders of magnitude.

The AMS-02 magnet is cooled by superfluid helium. There are two main reasons for this. Firstly, the specific latent heat and density of superfluid helium are both higher than in normal liquid helium. Since the amount of cryogen that can be carried is limited by the size of the helium vessel, this gives a useful endurance benefit (there is a greater mass of helium, and each kilogram has a higher cooling capability). 
Secondly, in zero gravity there can be no convection currents. In normal liquid helium this can result in thermal stratification, making it difficult to ensure that all parts of the system are fully cold. In the superfluid state, however, the very high thermal conductivity makes it impossible for the helium to support large temperature gradients, so the system remains isothermal.

Any large cryogenic vessel has to be viewed as a potential safety hazard, particularly when it is in an enclosed space such as the payload bay of the Space Shuttle. Safety of the AMS magnet has to be assured in ground handling operations, during launch, on orbit and during landing. All cryogenic volumes, as well as the vacuum tank, are protected by burst discs to prevent excessive pressures building up in any fault conditions. Some of the burst discs have to operate at temperatures below $2 \mathrm{~K}$, and these have been the subject of a special development and testing programme. In addition, extra protection is provided to mitigate the effect of a catastrophic loss of vacuum. This could be caused, during ground handling operations, by a serious rupture of the vacuum case. If the vacuum case had a large puncture, air could rush through the gap and condense on the surface of the helium vessel. This would result in rapid pressurisation and venting of the helium in the vessel. To slow down the rates of pressurisation and venting (making the pressure relief path smaller and more manageable) a $3 \mathrm{~mm}$ layer of lightweight cryogenic insulation will be applied to the outside surface of the helium vessel. Carefully constructed experiments have shown that this insulation reduces the heat flux to the superfluid helium by a factor of 8 following a sudden, total loss of vacuum. All parts of the AMS magnet system are subject to a battery of tests to ensure their quality and integrity, and their suitability for the mission.

Every one of the 14 superconducting coils will have been tested before assembly into the final magnet configuration. All twelve of the flux return coils have been tested successfully, and the first of the two dipole coils is undergoing final preparations for testing. A special test facility has been constructed which allows the coil to be operated under cryogenic conditions as close as possible to flight. In particular, the coil is mounted in a vacuum space, and is cooled by a thermal bus bar of the same construction as the one in the flight system.

A full-scale replica of the superfluid thermal bus bar system outlined above was designed and assembled.

Heaters on the part of the thermal bus bar outside the helium vessel were then used to simulate the heat load due to the magnet coils being charged. Up to $6 \mathrm{~W}$ could be generated by the heaters and transferred by Gorter-Mellink conduction through the thermal bus bar to be dissipated in the vessel of boiling helium. If the heaters were used to generate more power, the Gorter-Mellink conduction broke down: the thermal bus bar was unable to transfer the heat and the temperatures within it began to rise rapidly. These results corresponded to the calculations carried out before the tests.

A series of experiments has been carried out to determine what happens in the event either of a catastrophic loss of vacuum or of a much smaller vacuum leak. A test facility was designed and assembled in which either of these scenarios could be investigated on a small scale. The test facility contained a 12 litre helium vessel, together with a system of valves, sensors and high-speed data acquisition. To carry out a test, the 12 litre vessel was filled with superfluid helium at $1.8 \mathrm{~K}$. A fast-acting valve was then used to open a large hole in the insulating vacuum to simulate a catastrophic leak, or a very small hole to simulate a small leak. By monitoring the rise of pressure and temperature, and the rate of change of mass of the vessel, the heat flux and venting rate could be calculated. These results can be extrapolated directly to the AMS-02 magnet, and have been used to qualify the cryogenic system for flight on the Space Shuttle.

In addition to these investigations, tests have also been carried out on prototype burst discs. Discs for protecting the vacuum tank have undergone vibration testing followed by controlled bursts. These tests have shown that the discs are not affected by the levels of vibration encountered during a launch. Further tests have been carried out on discs for protecting the helium vessel, which operate at $1.8 \mathrm{~K}$. These discs have been shown to have extremely good leak tightness against superfluid helium. 


\section{The Transition Radiation Detector (TRD)}

Transition radiation [9] is the electromagnetic radiation that is emitted when charged particles transverse the boundary between two media with different dielectric properties $\varepsilon_{1}$ and $\varepsilon_{2}$. For the AMS-02, the Transition Radiation Detector (TRD) is realized by a $20 \mathrm{~mm}$ thick fleece which is used as radiator. A charged particle traversing this detector produces characteristic electromagnetic radiation depending on its mass and energy. Since the particle momentum is determined with the AMS-02 silicon tracker by measuring the trajectory inside the magnet, the detected transition radiation (TR) can be used to distinguish between particles of different masses, like positrons and protons.

The TR photons are detected in straw tubes, filled with a Xe:CO2 (80\%:20\%) gas mixture and operated at $1600 \mathrm{~V}$. With a probability of about $50 \%$ TR photons are produced in the $20 \mathrm{~mm}$ thick fleece and detected by the straw. The operation principle of TRDs is very well understood and they are used in large particle physics experiments (ATLAS [10] at CERN, HERA-B [11] at DESY). The new challenge is to operate such a large gas detector safely and reliably in space.

In total 328 TRD modules, each containing 16 straws, of lengths between 0.8 and $2 \mathrm{~m}$ are arranged in 20 layers each with $20 \mathrm{~mm}$ of $0.06 \mathrm{~g} / \mathrm{cm}^{3}$ polypropylene/polyethylene $10 \mu \mathrm{m}$ fiber fleece (Figure 4) The lower and upper four layers are oriented parallel to the AMS-02 magnetic field while the middle 12 layers run perpendicular to provide 3D tracking. They are supported by a conical octagon made of aluminumhoneycomb walls with carbon-fiber skins and bulkheads. A mechanical precision of better than $100 \mu \mathrm{m}$ assures gas gain homogeneity better than $1 \%$. The precision of the wire positioning was verified with a high-resolution computer-tomography-scan as shown and was found to be within $30 \mu \mathrm{m}$. On the outside of the conical octagon structure a grid structure (see Figure 5) built out of carbon fiber tubes, will be attached to support the services (gas tubes, HV cables, signal cables).

A coupled load analysis of the entire TRD structure [12] as been carried out by means of a detailed Finite Element Calculation. As the result of the modal analysis the first natural eigen-frequency turns out to be 54 $\mathrm{Hz}$, above the required $50 \mathrm{~Hz}$. Various experimental tests have been used to verify material properties and analytical calculations.

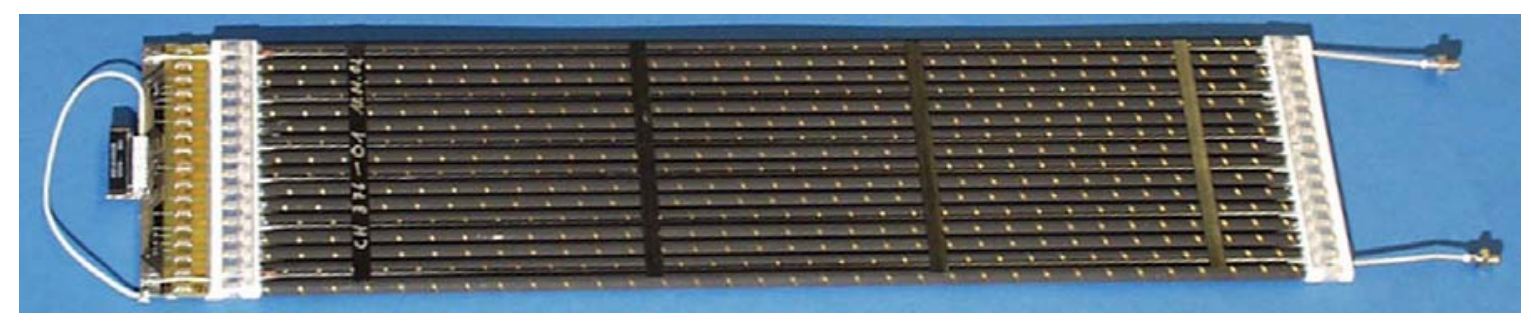

Figure 4: AMS-02 TRD module with 16 straw tubes 


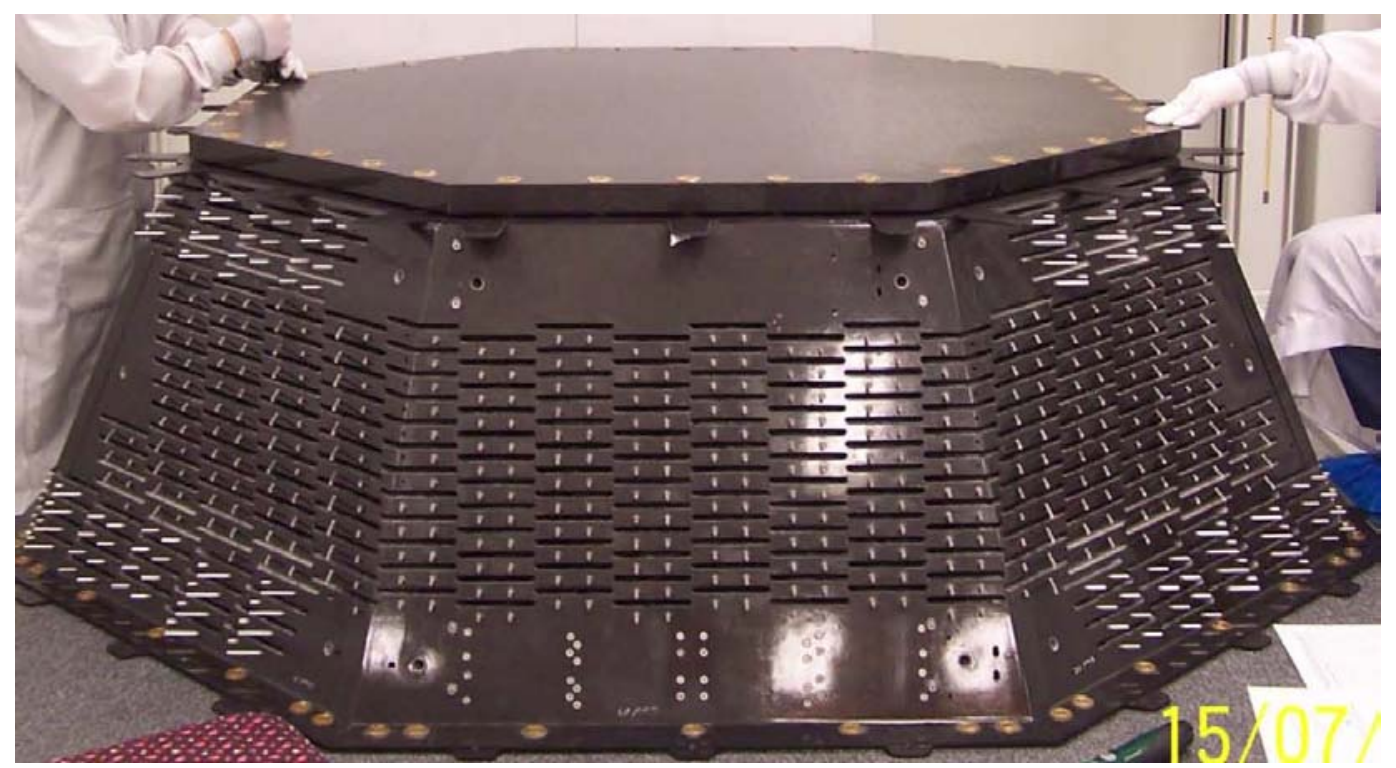

Figure 5. The TRD Octagon Support Structure

Table 2. Breakdown of the TRD weight

\begin{tabular}{|l|c|}
\hline Component & Weight [kg] \\
\hline Radiator & 60 \\
Straw Tube Modules & 54 \\
Octagon & 114 \\
Support, Shielding and Services & 100 \\
\hline Xe:CO $\mathrm{CO}_{2}$ & 54 \\
Gas System & 63 \\
Crate Electronics & 53 \\
\hline Total & $\mathbf{4 9 8}$ \\
\hline
\end{tabular}

The optimized AMS-02 TRD design with a diameter of $2.2 \mathrm{~m}$ and 5248 straw tubes arranged in 20 layers weighs less than $500 \mathrm{~kg}$ (see Table 2)

To separate protons from positrons, etc, anode signals from ionization losses alone have to be distinguished efficiently from signals containing both this energy deposit and absorbed TR photons with a pulse height readout. The signals of all straw tubes are digitized after a signal from the general AMS trigger and filtered in the first step of data reduction. Only good hits, defined as $3 \sigma$ above the noise level or about one tenth the signal left by a singly charged minimum ionizing particle (MIP), are sent to the higher levels system of AMS data acquisition (DAQ) system. The front end electronics is based on VA analog multiplexers [13] It is equipped with a serial 12-bit analog to digital converters (ADC) and covers a dynamic range of 40 MIPs (at a gas gain of 3000). The readout time, including the digitization of the 64 connected channels, is $87 \mu \mathrm{s}$. Altogether the electronics consumes less than $100 \mathrm{~W}$ to power, readout and control the 5248 channels of the TRD.

The AMS-02 TRD gas system is shown in Figure 6: includes the Xe and CO2 storage vessels with sufficient gas for the 3-5 year AMS-02 mission with a safety margin of four. It provides premixed gas from a limited transfer volume ( $\sim 1$ liter) under computer control daily through pumps and valves to control the 
gas circulation. It also monitors gas quality. Manifolds distribute the gas to the 5248 proportional wire tubes, which are arranged in 41 parallel segments. At all points, the valves have a two fold redundancy.

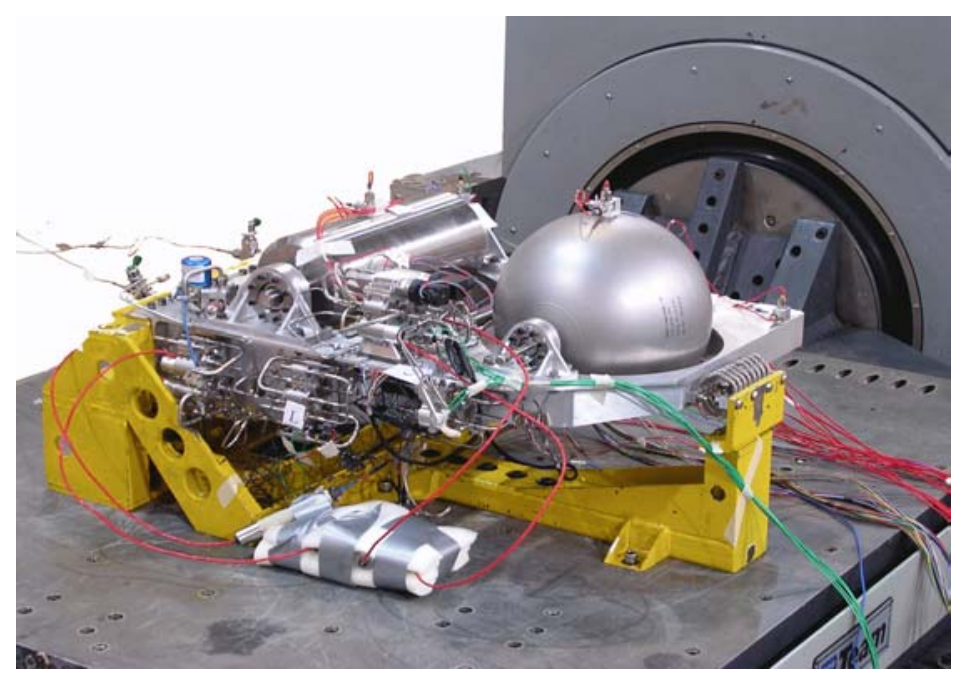

Figure 6: Box S engineering prototype and test fixture (yellow) mounted on slip table

To verify the proton rejection power of the AMS-02 TRD design a full 20 layer prototype was built with 40 modules of $40 \mathrm{~cm}$ in length arranged in two adjacent towers, equipped with VA analog multiplexer based front end electronics. This setup was used in the CERN T9, X7 and H6 beam lines to record 3 million tracks. For proton beam energies between 15 and $250 \mathrm{GeV}$ the proton rejection is well above 100 as shown in Figure 7.

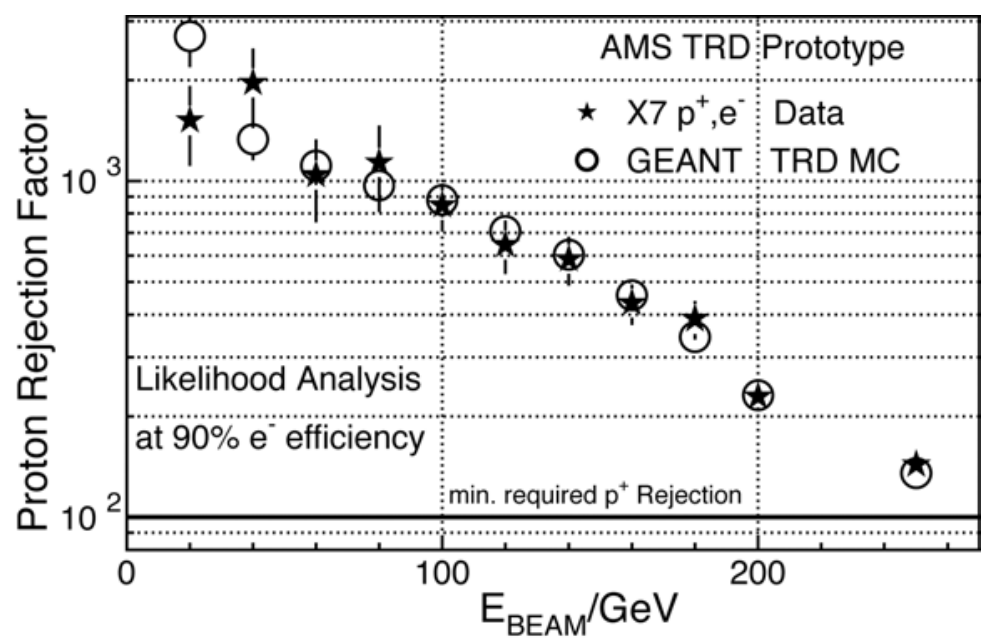

Figure 7: AMS-02 TRD proton rejection 


\section{The Time of Flight (ToF)}

Within AMS-02, the Time of Flight (ToF) scintillators provide the fast trigger for charged particles and converted photons, selection, at the trigger level, of particles within the main AMS acceptance, measurement of the particle velocity including the discrimination between upward and downward going particles and a measurement of the absolute charge which complements those made in the other detectors. The ToF system of the AMS-02 detector is based on previous well-established techniques [14] able to reach a precision in the time resolution of $100 \mathrm{ps}$. The design follows the experience gained with the AMS-01 detector [15], modified to take into account the different conditions in AMS-02, in particular, the stronger stray magnetic field at the photomultiplier tubes (PMTs).

The geometrical acceptance of the ToF, and consequently AMS-02 for charged particles, is $0.4 \mathrm{~m}^{2} \mathrm{sr}$, maximizing the sensitivity of the spectrometer in the search for antimatter and other phenomena. This matches the acceptance of the silicon tracker within the magnet. The ToF system is composed of four roughly circular planes of $12 \mathrm{~cm}$ wide scintillator paddles, one pair of planes above the magnet, the upper TOF, and one pair below, the lower ToF (Figure 8). Each paddle is instrumented with two PMTs at each end.

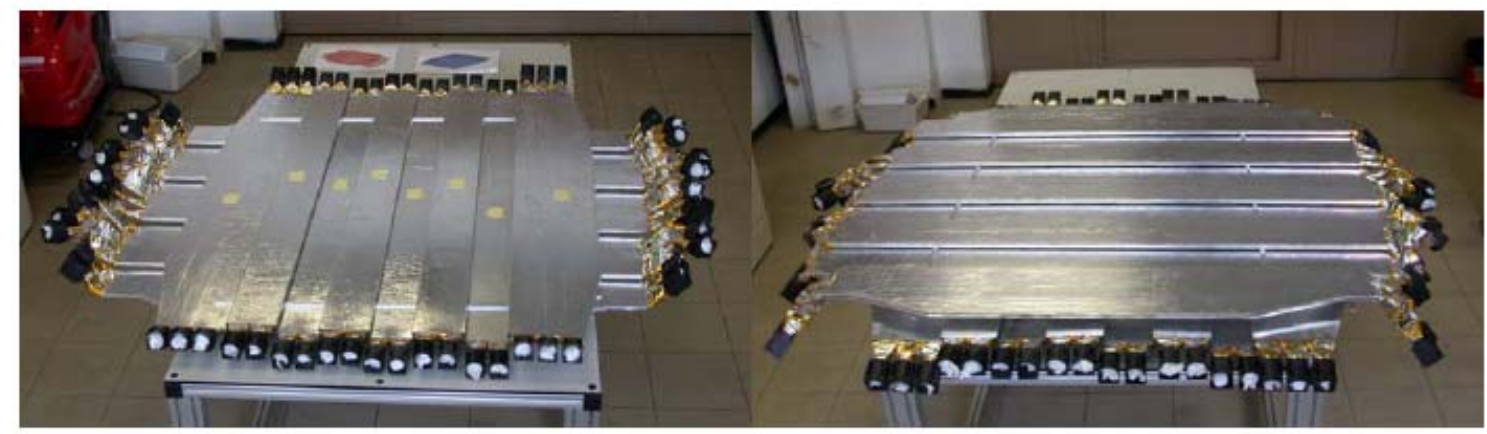

Figure 8: Flight paddles during the assembly of the upper (left) and lower (right) TOF

The ToF system provides a very fast and reliable response to the energy loss of charged particles, in order to provide the general data acquisition system (DAQ) with the fast trigger signal (FT), that is used as the reference time for the event. The overlapping and crossed paddle geometry of the AMS-02 ToF allows a granularity of about $12 \times 12 \mathrm{~cm} 2$ for trigger purposes with $\sim 100 \%$ efficiency and a gate of $50 \mathrm{~ns}$.

The resolution in the time of flight needed to satisfy the physics requirements is $120 \mathrm{ps}$. The scintillator paddles are $1 \mathrm{~cm}$ thick, a compromise between minimum thickness and the light output needed to reach this resolution. Downward going charged particles are distinguished from upward going at the level of $10^{9}$.

The system measures the energy loss by a charged particle (to first order proportional to the square of the particle charge) with a resolution sufficient to distinguish nuclei up to charge $Z \sim 20$. The system guarantees redundancy, with two PMTs on each end of the paddles and double redundant electronics and can operate in vacuum over the temperature range -20 to $+50{ }^{\circ} \mathrm{C}$. It has a weight of less than $280 \mathrm{~kg}$ and a power consumption, including all electronics, of less than $170 \mathrm{~W}$. System components have been qualified for use in space and have been tested with particle beams.

The ToF counters must operate in the stray field of the AMS-02 cryomagnet, which ranges up to $3 \mathrm{kG}$. A thorough investigation selected a PMT which can operate under these conditions - the Hamamatsu R5946 - 
provided the PMT axis is aligned within 45 degrees of the field direction. This has been a primary design consideration in the construction of the TOF system. Each of the four planes is composed of 8 or 10 paddles. In total, the ToF detector is instrumented with 144 Hamamatsu R5946 PMTs, with a bialkali photocathode, a borosilicate glass window and 16 bialkali dynodes. The spectral response ranges from 300 to $600 \mathrm{~nm}$ with a maximum response at $420 \mathrm{~nm}$ (corresponding to a quantum efficiency of about $20 \%$ ), which matches the scintillator output.

The PMTs are mounted in a polycarbonate housing which also contains an optical joint between the conical light guide and the PMT window and an $80 \mathrm{M}$. resistor HV divider attached to the base of the PMT. The divider and base are completely potted to avoid corona effects. The TOF system must be able to operate in space for years without human intervention and with temperatures ranging from -20 to $+50{ }^{\circ} \mathrm{C}$. In addition, the detector must survive the strong accelerations produced by the shuttle launch, and its performance should not be affected by this vibration. Hence, a series of thermal-vacuum and mechanical tests have been carried out on the ToF system, in addition to the tests on the full AMS-02 detector before flight.

The ToF counters were tested in ion beams at CERN in 2002 and again in 2003. The beams were obtained by the fragmentation of the primary SPS $\mathrm{Pb}$ beam at 20 and $158 \mathrm{GeV} / \mathrm{c} / \mathrm{A}$ against a $\mathrm{Be}$ or $\mathrm{Pb}$ target, within different momentum per nucleon windows using the H8 selection line. In 2002 the response of the counters were compared to one of the AMS-01 counters. In 2003, four counters with different configurations of the light guides were tested.

The time of flight measurement between the C2 and C3 counters is shown in Figure 9 as a function of the particle charge. As the measurement in AMS-02 will be done with four independent measurements, the time resolution which can be inferred is of the order of $130 \mathrm{ps}$ for a MIP.

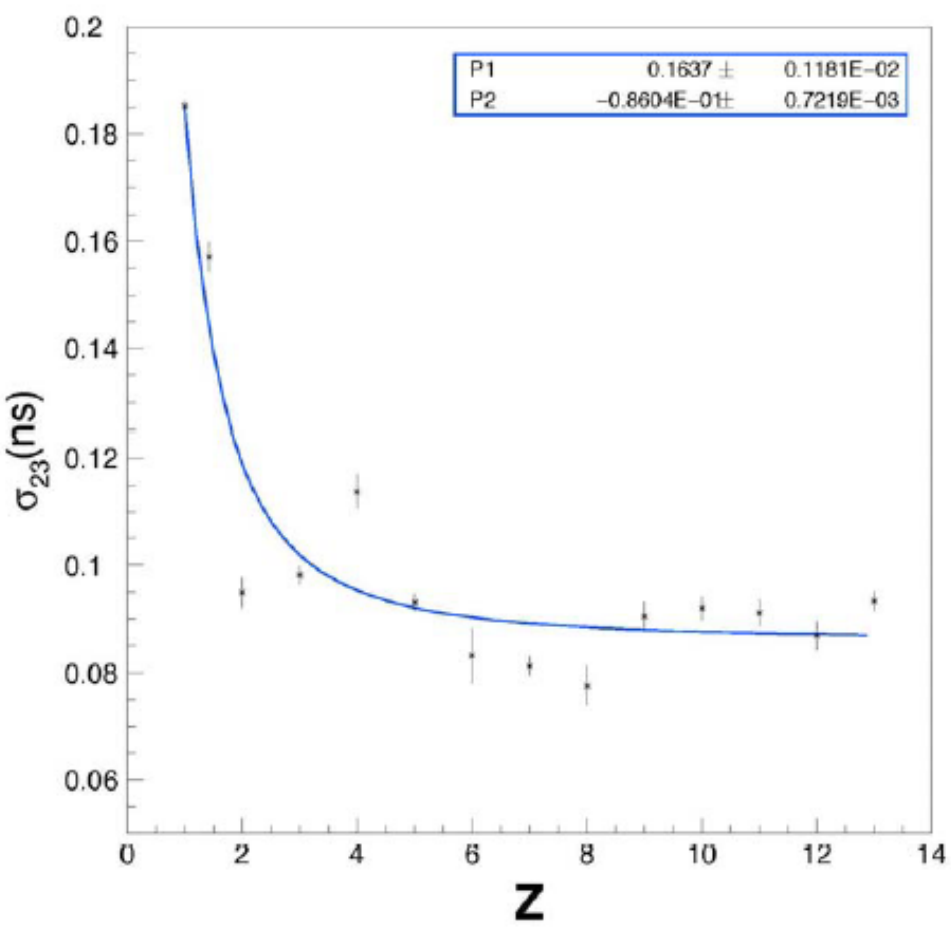

Figure 9: Time of Flight Resolution with two planes vs. particle charge 


\section{The Silicon Tracker}

The AMS-01 Silicon Tracker [16] was the first application in space of the high precision silicon technology developed for position measurements in accelerator experiments [17]. The high modularity, low voltage levels $(<100 \mathrm{~V})$, and gas-free operation of the device is well suited to operation in space. The 1998 shuttle test flight demonstrated both the successful adaptation of the technology to the space environment and the feasibility of large area detectors. Silicon micro-strip sensors were originally developed for vertex detectors in colliding-beam experiments in order to provide a few high precision position measurements near the interaction point. The AMS application differs considerably. The tracking information is provided uniquely by the silicon sensors, which implies a large surface area and higher inter-strip capacitances. The major challenges were to maintain the required mechanical precision and low-noise performance in the large scale application, and to do so in outer space.

The silicon tracker is composed of $41.360 \times 72.045 \times 0.300 \mathrm{~mm} 3$ double-sided silicon micro-strip sensors. The sensors have been produced at silicon foundries located in Switzerland [18] and Italy [19] using identical geometries and processing procedures. Over 4000 sensors have been produced to select the $\sim 2500$ sensors of the very high quality required to assemble the Silicon Tracker units (ladders). Over $2 \times 10^{7}$ electrical measurements have been performed using four automatic test stations. This large number of sensors makes the AMS Silicon Tracker the largest precision tracking detector ever built for a space application. Figure 10 shows the principal elements of the silicon ladder and the main components of the readout hybrids.

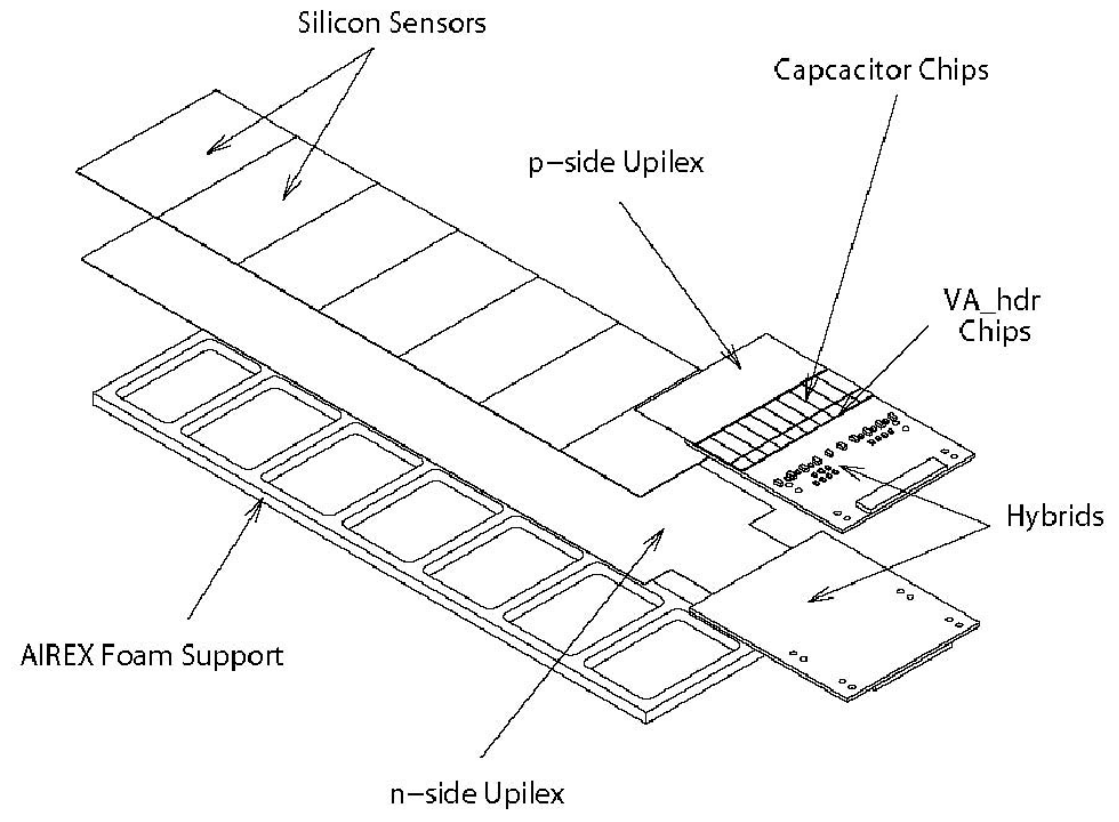

Figure 10: The principal components of the silicon ladder

The principal goals of the ladder fabrication are to guarantee the required precision for the relative alignment of the silicon sensors $(<5 \mu \mathrm{m})$, and minimize the degradation of the electrical performance due to handling and ultra-sonic bonding. Ladder fabrication was organized between three centers operating with identical 
procedures derived from the AMS-01. During fabrication the sensor positions on a ladder are recorded with a 3D semiautomatic measuring machine. The results for the sensor alignment for the first 125 (out of 192) AMS-02 ladders is better than $5 \mu \mathrm{m}$ rms.

The tracker support structure is divided into three sections: a carbon fiber cylindrical shell which supports the planes 2 to 4 located inside the magnet, and two carbon fiber flanges which support the exterior planes 1 and 5 [20]. With respect to the AMS-01 configuration, the number of silicon layers has been increased from 6 to 8 by suppressing one internal plane and equipping both sides of the remaining three internal planes with silicon ladders. When ladders equipping a full tracker plane are produced, they are integrated onto the corresponding support plane. Supervision, quality control and traceability are ensured by a database developed for that purpose. The activities start with acceptance tests of the ladders arriving from all production lines and then proceed in steps toward integration of the complete detector. Installation of the second layer on the other side is more delicate since one has access from only one side for fixation. The assembled plane is finally stored in a light-tight container under dry nitrogen. Figure 11 shows the first fully equipped inner plane.

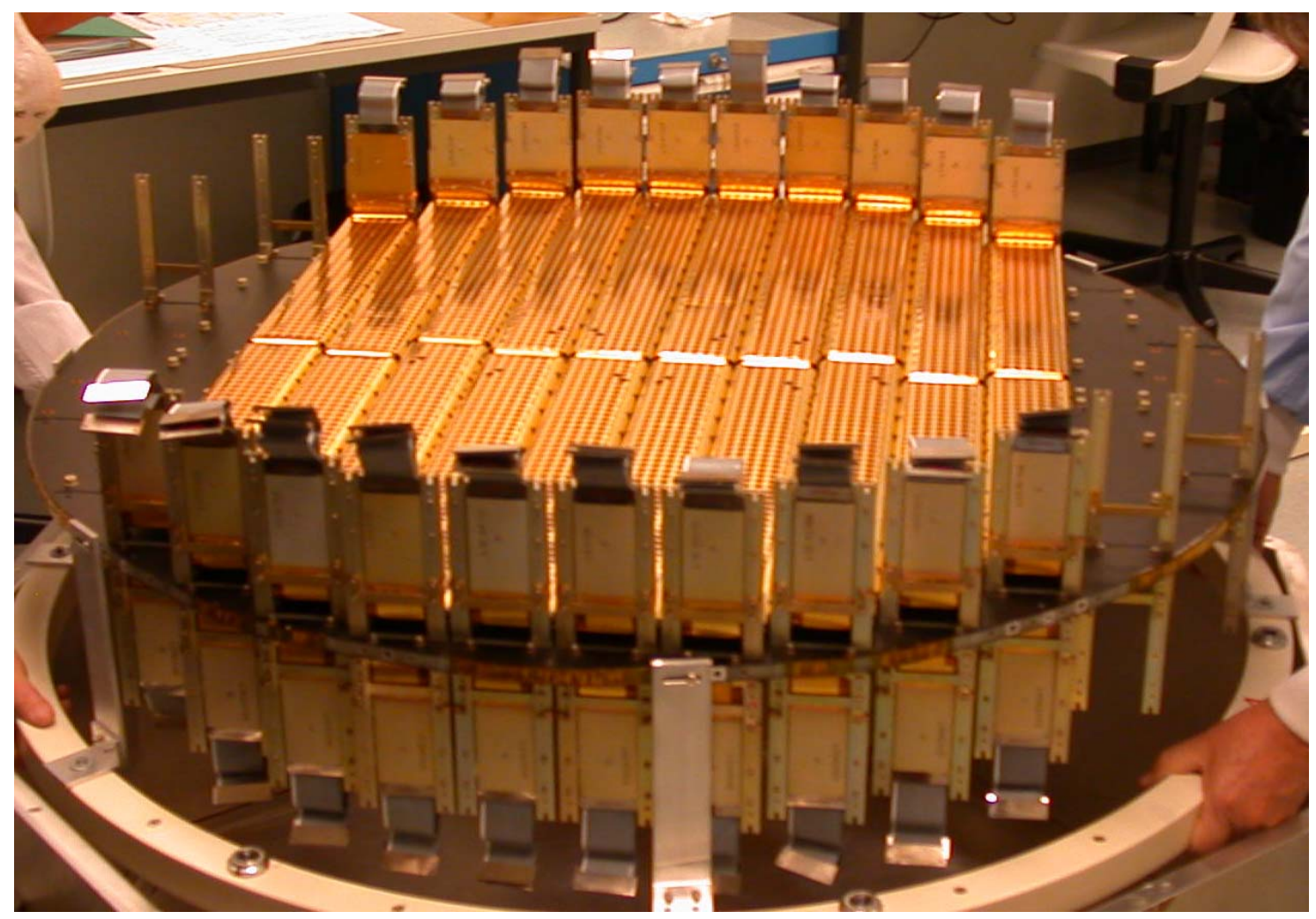

Figure 11: Plane numbers 2 completely equipped with ladders

The silicon sensors are grouped together, for readout and biasing, in ladders of different lengths to match the cylindrical geometry of the AMS magnet. The maximum combined strip length in the silicon for a single readout channel is $60 \mathrm{~cm}$. The relatively large input capacitance $(30-180 \mathrm{pF})$, as well as the need for a high dynamic range $(<100$ MIPs), led to the development of a new front-end readout chip based on the low-noise Viking design, the VA_hdr7 [21]. 
Each of the 64 channels of the VA_hdr chip consists of a charge sensitive amplifier, a CR-RC semiGaussian shaper, and a sample-and-hold stage. An analog multiplexer, shift register and buffer are incorporated in the chip for sequential data output at a maximum clock frequency of $10 \mathrm{MHz}$. The equivalent noise charge as a function of capacitance load Cdet has been measured to be $(350+4 \mathrm{Cdet} / \mathrm{pF}) \mathrm{e}^{-}$at a $6 \mu$ s peaking time and nominal bias currents. The VA_hdr chips have an average power consumption 0.7 $\mathrm{mW}$ per channel. The single channel response of the VA_hdr chip has been measured to be linear up to $\sim 75$ MIPs. The strips of the silicon sensors are ac-coupled to the VA_hdr via $700 \mathrm{pF}$ capacitor chips.

The hybrids are mounted on carbon fiber-metal cooling bars, which evacuate the heat generated by the frontend electronics inside the magnet. The presence of the superconducting magnet requires an active cooling system for the tracker. The AMS-02 Tracker Thermal Control System (TTCS) is a two-phase, mechanically pumped loop system. The cooling liquid, $\mathrm{CO} 2$ at about 80 bar pressure, is circulated by a pump. Outside of the tracker volume, the fluid passes through a heat exchanger to keep the incoming fluid just at the boiling point while minimizing the pre-heater power required. It is then directed to condensers on the tracker thermal wake and ram radiator panels facing deep space. There, the vapor/liquid mixture is cooled to below the boiling point, and then returns to the pump input, closing the circuit. Ammonia heat pipes embedded in the radiators increase their effectiveness. The relative flow of fluid to the wake and ram radiators is selfadjusting, the fluid will preferentially flow towards the cooler radiator.

Space based particle detection systems have to cope with a far wider range of environmental conditions than those at accelerators. This concerns notably the vibrations during the transport before deployment and the rapid periodic changes in the thermal settings due to solar radiation and cooling while in the shadow of Earth. With the AMS-02 silicon tracker, charged particle tracks are traced at 8 space points in a $\sim 1 \mathrm{~m}^{3}$ sized B-field to an accuracy of better than $10 \mu \mathrm{m}$. The alignment system provides optically generated signals in the 8 layers of the silicon tracker that mimic straight (infinite rigidity) tracks. The AMS-02 tracker is equipped with $2 \times 10$ pairs of alignment control beams. The beams are narrow (diameter $<0.5 \mathrm{~mm}$ ) and of small divergence $(<1 \mathrm{mrad})$. The AMS approach to silicon tracker alignment control using IR laser beams fulfills the requirements of a space borne experiment. It is light weight $(3 \mathrm{~kg})$, low power $(1 \mathrm{~mW})$, low dead time $(<1 \%)$ and provides a precision exceeding the tracker resolution $(8 \mu \mathrm{m})$ with less than 100 laser shots. The success of the AMS approach in Si tracker alignment control by IR laser beams has lead the team building the largest Si tracker array [22] to develop a similar system for 10 years of operation at the LHC.

An extensive series of tests have been performed to verify the performance of the AMS-02 silicon tracker. For what concerns the spatial resolution the residual distributions of the ladder, described by a Gaussian function and flat background., give widths of the Gaussians of 8.5 and $30 \mu \mathrm{m}$ respectively for the p- and n-sides. To study the AMS-02 ladder response to light and heavy ions, six ladders were exposed to an ion beam at CERN in October 2003. The tracker $\mathrm{Z}$ measurements are compared to those of prototype RICH detector in Figure 12. An excellent correlation to the RICH Z measurement is seen for both side of the silicon sensors, namely the $\mathrm{K}$ and the $\mathrm{S}$ side, respectively.

If compared to the precursor AMS-01 mission, the performance of the tracker in terms of signal to noise, position resolution and charge resolution has been greatly enhanced. The increase of silicon layers from 6 to 8 , together with the more powerful AMS-02 cryomagnet, has significantly increased the physics reach of the AMS-02 detector, making a wide range of physical phenomena accessible during the AMS-02 mission. 

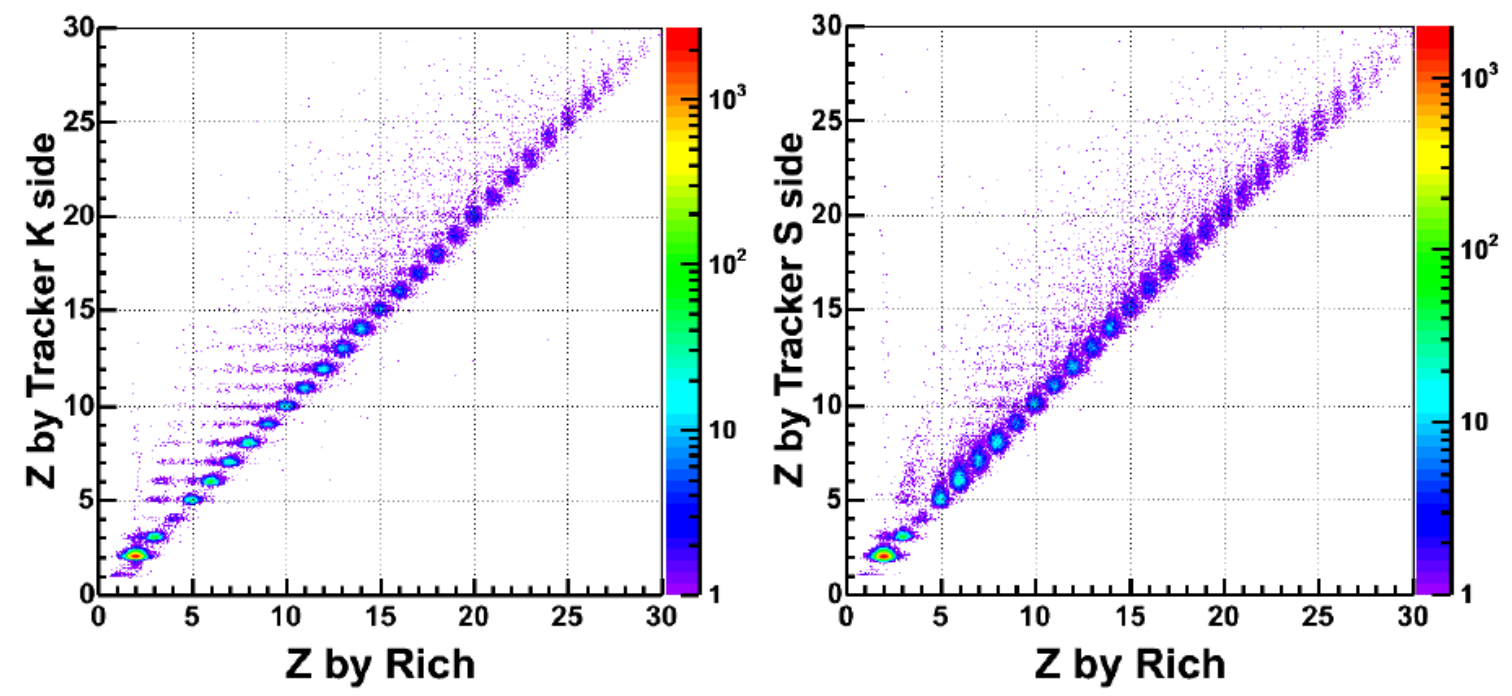

Figure 12: Comparisons of charge $(\mathrm{Z})$ measurements by the Tracker and $\mathrm{RICH}$

\section{The Ring Imaging Cerenkov Detector (RICH)}

In order to study fundamental topics in astroparticle physics such as the existence of antimatter or the relative abundances of light isotopes and charged nuclei, it is necessary to have a precise determination of the masses of charged particles. The mass of a particle is related to its momentum, $p$, and velocity, $\beta$, through the expression $\quad m=(p / \beta) \sqrt{ }(1-\beta)^{2}$ and its determination is based on the measurement of both quantities. In the AMS spectrometer, the momentum is determined from the information provided by the Silicon Tracker with a relative accuracy of $\mathrm{O}(1 \%)$ over a wide range of energies. This entails an error of the same order on the mass of the particle so, in order to overcome this uncertainty, the velocity has to be measured with a relative accuracy of about 1 per mil. For this purpose a Ring Imaging Cerenkov Detector $(\mathrm{RICH})$ has been designed with a large geometrical acceptance to operate in the environmental conditions of the outer space.

Within AMS-02 the RICH is placed on the lower part of the spectrometer, between the lower Time of Flight Counters and the Electromagnetic Calorimeter (Figure 13). It has a truncated conical shape with $60 \mathrm{~cm}$ upper radius, $67 \mathrm{~cm}$ lower radius, a height of $47 \mathrm{~cm}$ and a supporting plate on the top that holds a $3 \mathrm{~cm}$ thickness layer of dielectric material (radiator). The lower plane, which has a $64 \times 64 \mathrm{~cm}^{2}$ square central hole to let particles go unaffected to the Electromagnetic Calorimeter, supports an array of 680 light guides and photomultipliers as well as the front-end electronic boards. A charged particle coming from above first crosses the radiator creating a cone of Cerenkov radiation. Most of the photons go directly to the photon detectors in the lower plane. The other photons are reflected on the lateral surface of the cone and also directed to the photon detectors. From the spatial coordinates of the photomultiplier pixels and the direction of the incoming particle, the Cerenkov cone is reconstructed and the velocity of the particle determined. Upward going particles do not leave this signal and can thus be unambiguously discriminated. 


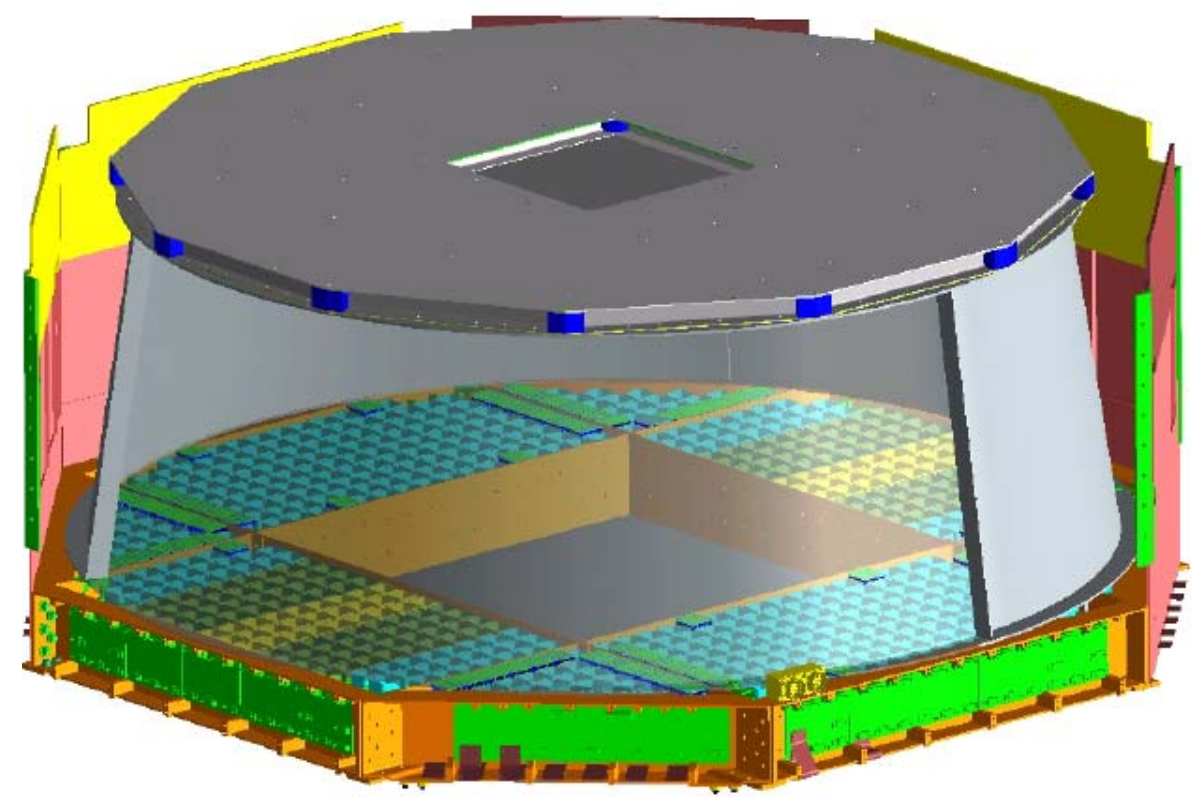

Figure 13: Exploded view of the Ring Imaging Cerenkov Detector (RICH)

Several dielectric materials have been considered as possible radiators. Their performance has been analysed by a Monte Carlo simulation and, for the most appropriate ones, tests have been performed with ion beams at CERN and cosmic rays at LPSC (Grenoble). The material that has shown better performance for the experimental goals are silica Aerogels. These compounds are a mixture of $m(\mathrm{SiO} 2)$ and $2 m(\mathrm{H} 2 \mathrm{O})$, with $m$ an integer, and have a porous structure with bubbles, most of them of small size compared to optical wavelengths. The refractive index is an average between the ones of air and the mixture and has an almost linear dependence with the density. About $30 \%$ of the Cerenkov photons emerging from the radiator are pointed outside of the photon detector array. A reflector has been designed to collect those photons in the array. It consists of a supporting structure with a truncated conical shape of 134 and $114 \mathrm{~cm}$ lower and upper diameters and a thin film of reflective coating deposited on the inner surface. In order to maintain the 1 per mil velocity resolution, it has been designed with strict requirements on the tolerances as shown in Table 2.

Table 2: Parameters and designed tolerances for the reflector

\begin{tabular}{|l|c|}
\hline Conicity & $200 \mu \mathrm{m}$ \\
\hline Centering & $100 \mu \mathrm{m}$ \\
\hline Reflectivity & $\begin{array}{c}>85 \%(\text { at } \lambda=420 \\
\mathrm{nm})\end{array}$ \\
\hline Roughness & $<150 \mathrm{~nm}$ \\
\hline Slope error & $<1 \mathrm{mrad}$ \\
\hline
\end{tabular}

The Cerenkov photons are collected at the bottom of the detector by an array of 680 photomultiplier tubes (PMTs) that withstand moderate magnetic fields. However, the residual field of the superconducting magnet in the region where the photomultipliers are placed is $>300 \mathrm{G}$ so they have to be surrounded by a shielding case and, as a consequence, a light collection system has to be designed in order to direct the Cerenkov photons from the top of the shielding case to the sensitive part of the photomultipliers. Most appropriate for the characteristics of this detector is the HAMAMATSU R7600-00-M16 due to then reduced size, fast response under low operational voltage $(800 \mathrm{~V})$, large anode uniformity and low sensitivity to external 
magnetic fields. These photomultipliers have a multianodic structure with $4 \mathrm{x} 4$ pixels, each one with a sensitive zone of $4 \times 4 \mathrm{~mm}^{2}$. This guarantees a resolution in the measurement of the velocity of 1 per mil. These PMTs also have a high quantum efficiency $(\sim 20 \%)$ in a range of wavelengths between 300 and 600 $\mathrm{nm}$, well within the optical region of the radiator and where the refractive index is, to a large extent, independent of the photon energy. With this efficiency, for an ultra relativistic particle of charge $Z=1$, an average of 8 photons are detected for a radiator with a refractive index of $n=1.03$. Each photomultiplier has $4 \times 4$ pixels and every light-guide structure has 16 independent units with 4 different shapes and fills a total volume of $13 \mathrm{~cm} 3$. The optimum dimensions have been determined to maximise the photon collection efficiency. A Monte Carlo simulation of different physical processes has shown that sufficient Cerenkov photons are collected to achieve a resolution in the velocity of charged particles of $\sigma_{\beta} / \beta \sim 10^{-3}$.

Complete cells were tested on a vibration table to ensure that the structure will withstand the shuttle take-off. In order to validate the design of the AMS- $02 \mathrm{RICH}$, a prototype with an array of $9 \times 11$ cells similar to those of the final model has been constructed. The performance of this prototype has been tested with cosmic muons and, in October 2002, it was placed in a beam of secondary ions at the CERN SPS produced by the fragmentation of a $\mathrm{Pb}$ beam. The rigidity settings for the fragments on the transport line allow an effective selection of the secondary ions with specific values of the mass over charge ratio. In particular, beams of protons with rigidities between $5 \mathrm{GV}$ and $13 \mathrm{GV}$ and $\mathrm{A} / \mathrm{Z}=2$ ions with a rigidity of $40 \mathrm{GV}$ were selected. The rigidities were known with an accuracy of $1.5 \%$. The measured distribution of charges in the beam is shown in Figure 14 where the structure of individual ion peaks up to $Z=26(\mathrm{Fe})$ is clearly visible (protons have been suppressed).

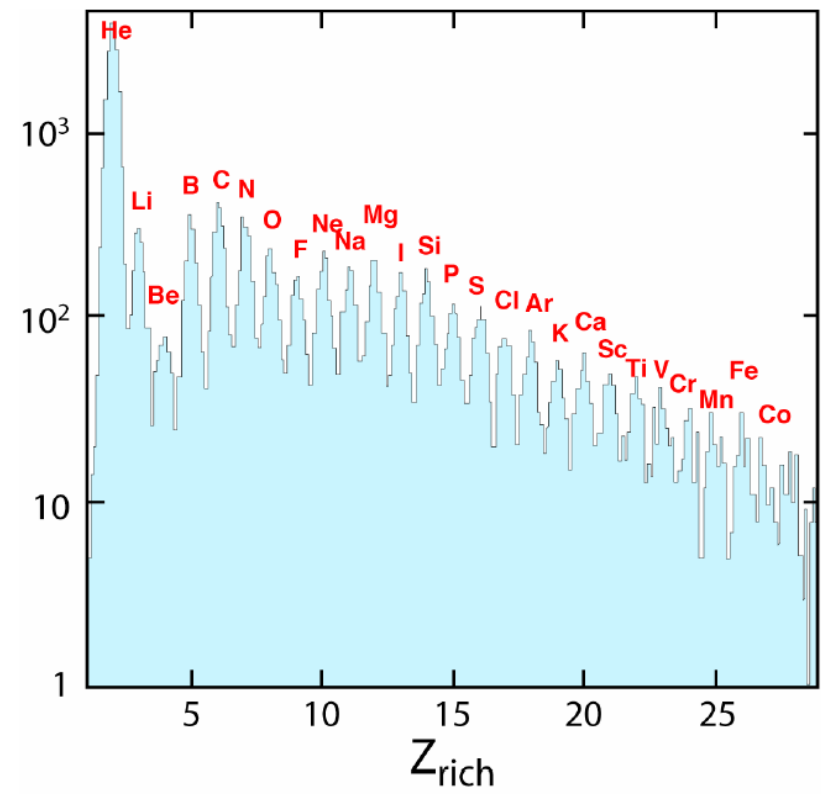

Figure 14: Measured distribution of charges $(\mathrm{Z}>1)$ for the $\mathrm{CIN} n=1.03$ radiator

\section{The Electromagnetic Calorimeter (ECAL)}

The Electromagnetic Calorimeter (ECAL) of the AMS-02 experiment is a fine grained lead-scintillating fiber sampling calorimeter [23] that allows precise, 3-dimensional imaging of the longitudinal and lateral shower development, providing high $\left(>10^{6}\right)$ electron/hadron discrimination in combination with the other AMS-02 detectors [24] and good energy resolution. The calorimeter also provides a standalone photon 
trigger capability to AMS. The mechanical assembly has met the challenges of supporting the intrinsically dense calorimeter during launch and landing with minimum weight. The light collection system and electronics are optimized for the calorimeter to measure electromagnetic particles over a wide energy range, from $\mathrm{GeV}$ up to TeV. A full-scale ECAL qualification model was tested using electrons and protons beams with energies ranging from 3 to $180 \mathrm{GeV}$.

The AMS-02 ECAL has an active area of $648 \times 648 \mathrm{~mm}^{2}$ and a thickness of $166.5 \mathrm{~mm}$. The calorimeter is composed from "superlayers", each $18.5 \mathrm{~mm}$ thick and made of 11 grooved, $1 \mathrm{~mm}$ thick lead foils interleaved with layers of $1 \mathrm{~mm}$ diameter scintillating fibers and glued together with epoxy. It has a relative lead-fiber-glue volume composition of 1:0.57:0.15 and an average density of $6.9 \pm 0.2 \mathrm{~g} / \mathrm{cm}^{3}$.

In each superlayer, fibers run in one direction only. The detector imaging capability is obtained by stacking superlayers with fibers alternatively parallel to the x-axis (4 layers) and y-axis (5 layers). The calorimeter has a total weight of $496 \mathrm{~kg}$ and a thickness corresponding to about 17 radiation lengths.

Fibers are read out, on one end only, by four anode Hamamatsu R7600-00-M4 photomultipliers (PMTs); each anode covers an active area of $9 \times 9 \mathrm{~mm}^{2}$, corresponding to 35 fibers, defined as a cell. In total the ECAL is subdivided into 1296 cells (324 PMTs) and this allows a sampling of the longitudinal shower profile by 18 independent measurements.

The ECAL mechanical assembly (Figure 15), supports the calorimeter PMTs and attached electronics. It is designed to minimum weight with a first resonance frequency above $50 \mathrm{~Hz}$, a capability to withstand accelerations up to $14 \mathrm{~g}$ in any direction and thermal insulation limiting the gradient (the external temperature ranges from -40 to $+50{ }^{\circ} \mathrm{C}$ )

The optimization of the mechanical structure through finite element studies led to an aluminum alloy support frame, composed of four lateral panels which contain the PMTs and top and bottom honeycomb plates. The structural analysis was cross checked with a full scale, flight like qualification model. Sine-sweep and sineburst data and stress measurements showed excellent agreement with the ECAL finite element model. In particular, the first resonance frequency was found to be higher than $60 \mathrm{~Hz}$.

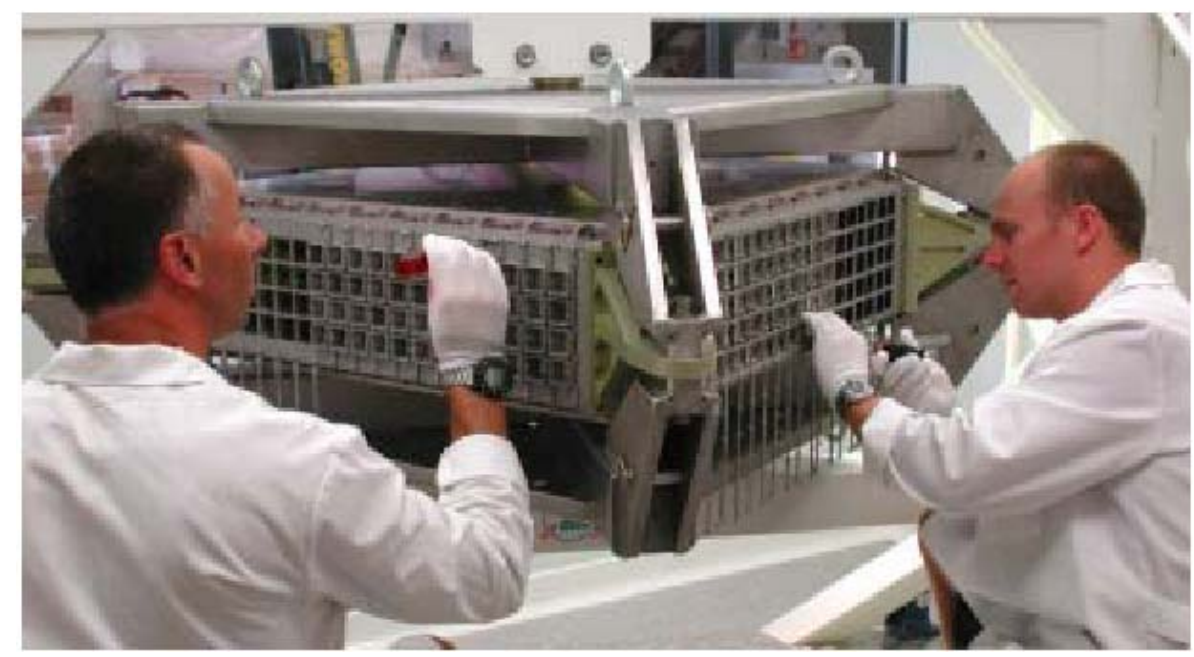

Figure 15: ECAL mechanical support structure 
Each superlayer is read out by 36 PMTs, arranged alternately on the two opposite ends in order to read out each fiber (no dead areas). Each PMT is surrounded by a magnetic shield which also contains light guides and the PMT base and front end (FE) electronics. To maximize light yield and reduce cross-talk between cells, photons from the fibers are collected by means of individual Plexiglas light guides. They are wrapped in aluminum foil with Chromium and quartz coating and inserted inside a polycarbonate support tube. Silicone optical joints are positioned on both ends to ensure good optical transmission from the fibers to the PMTs, even when high mechanical stresses are applied. To shield the residual magnetic field below $20 \mathrm{G}$, based on finite element calculations and measurements, the PMTs are positioned inside $1 \mathrm{~mm}$ thick soft iron square tubes with $30.5 \mathrm{~mm}$ sides and a length of $74 \mathrm{~mm}$.

The excellent imaging capabilities of the AMS-02 electromagnetic calorimeter and the good energy resolution provided by the last dynode signals, allow the implementation of a very efficient standalone trigger for photons with energies down to $2 \mathrm{GeV}$. The expected ECAL trigger efficiency in AMS-02 for unconverted photons of different energies is $90 \%$ at $2 \mathrm{GeV}$ and more than $99 \%$ for energies larger than 10 $\mathrm{GeV}$.

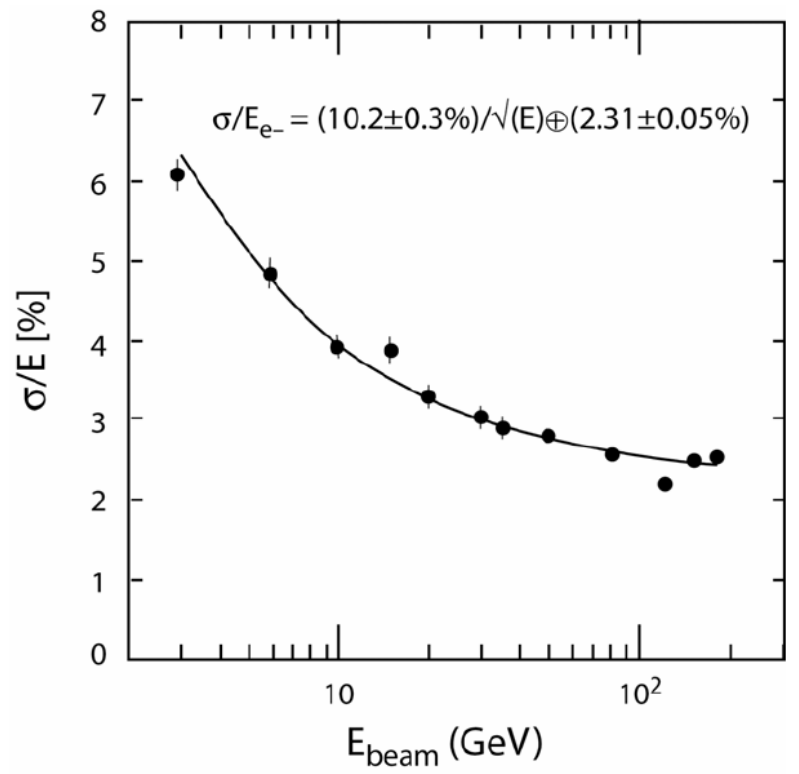

Figure 16: ECAL Energy resolution

In July 2002, the ECAL qualification model was exposed to the CERN SPS beam line H6A with muons, 120 $\mathrm{GeV}$ protons and antiprotons and 3 to $180 \mathrm{GeV} \mathrm{e}^{ \pm}$. From the fits to the ECAL response at different energies good linearity was found up to $40 \mathrm{GeV}$ (when longitudinal leakage is negligible), while deviations of the order of $5 \%$ at $80 \mathrm{GeV}$ and of $13 \%$ at $180 \mathrm{GeV}$ were observed. A leakage correction, based on the energy deposit in the last ECAL superlayer, was applied in order to estimate the total energy. The calorimeter energy resolution is shown in Figure 16, where the fractional uncertainty in the energy measurement, $\sigma(E) / E$, is plotted as a function of the nominal beam energy, $E$ (in GeV). The energy resolution is well parametrized by $\sigma(\mathrm{E}) / \mathrm{E}=(10.2 \pm 0.3) \% / \sqrt{\mathrm{E}}(\mathrm{GeV}) \oplus(2.3 \pm 0.1) \%$. This result was obtained for electrons entering ECAL at the center of the equipped region and is in good agreement with the Monte Carlo taking into consideration the errors due to the incomplete acceptance of the lateral shower development and to the calibration procedure. The ECAL discrimination power is about $1 / 250$ with an efficiency of $90 \%$; the rejection can be improved by combining Tracker and ECAL information to determine the energy/momentum ratio $(\mathrm{E} / \mathrm{p})$. To evaluate the effectiveness of this procedure with the test beam data, the momentum was 
smeared according to the Tracker resolution and the matching requirement was applied with a cut E/p $>0.8$, yielding an additional factor of 20 improvement in the discrimination power.

\section{Conclusions}

AMS-02 is the first precision particle physics experiment on the International Space Station. With its acceptance, resolution and ability to study different particles and nuclei over 3 to 5 years of data taking, it provides an opportunity to accurately explore new regions of physics.

Acknowledgements: the author thanks M. Capell for its support during the preparation of this paper. This work has been partly supported by the Italian Space Agency (ASI) contract I/021/05/0.

\section{References}

[1] G. Jungman et al., Phys. Rep. 267 (1996) 195;

J.R. Ellis et al., Nucl. Phys. B214, (1998) 3;

E.A. Baltz and J. Edsjo, Phys. Rev. D59 (1999) 23511;

T. Moroi and L. Randall, Nuc. Phys. B570 (2000) 455.

[2] P. Salati, et al., Nucl.Phys.Proc.Suppl. 81 (2000) 37.

[3] A.D. Dolgov, Phys. Reports 222 (1992) 309;

[4] J.G. Asbury et al., Phys. Rev. Lett. 18, 2 (1967) 65;

[5] J.J. Aubert et al., Phys. Rev. Lett. 33, 23 (1974) 1404.

[6] For example, L3 Collaboration, Nucl. Instr. Meth. A 289 (1990) 35.

[7] B. Blau et al., Grav. and Cosm.5 (2000), Suppl. pp. 1; B. Blau et al., IEEE Trans. on App. Sup. 12 (2002) 349.

[8] S. Harrison, et al., IEEE Trans. on App. Sup. Vol. 13 pp. 1381-1384.

[9] I. Frank, V. Ginzburg, J. Phys. 9 (1945) 353.

[10] ATLAS TDR 5, Vol.2, CERN/LHCC/97-17.

[11] HERA-B, DESY-PRC 00/04, 2000.

[12] AMS-02 Structural Verification Plan, JSC-28792, Rev. C.

[13] F. Hauler, Conf. Proc. IEEE NSS 2003, sub. IEEE NS.

[14] D. Bollini et al., Nuovo Cimento 61A (1969) 125;

A. Zichichi, Annals of Physics 66 (1971) 405;

M. Basile et al., Nucl. Instrum. and Meth. 179 (1981) 477.

[15] D. Alvisi et al., Nucl. Instrum. and Meth. A 437 (1999), 212.

[16] R. Battiston Nucl.Phys.Proc.Suppl. 44 (1995) 274;

J. Alcaraz et al., Nuovo Cim. 112A (1999) 1325;

W.J. Burger, Nucl.Phys.Proc.Suppl. 113 (2002)139.

[17] M. Acciarri et al., Nucl. Instr. and Method. A 351 (1994) 300.

G.F. Dalla Betta, et al., Nucl. Instr. and Method. A 431 (1999) 83.

[18] Colibrys SA, Maladière 83, CH-2007 Neuchâtel.

[19] ITC-irst; Via Sommarive 18, I-38050 Povo.

[20] Oerlikon Contraves; Birchstrasse 155, CH-8050 Zürich.

[21] IDE AS; Veritasveien 9, N-1323 Hovik.

[22] Ostaptchouk et al., 9 May 2001, CMS Note 2001/053, CMS - CERN.

[23] F. Cervelli et al., Nucl. Instr. and Method. A 490 (2002) 132; F. Cadoux et al., Nuclear Physics B (Proc. Suppl.) 113 (2002) 159.

[24] E.Choumilov et al., Nucl. Instr. and Method. A 426 (1999) 625. 
